\title{
Electromagnetic radiation from laser wakefields in underdense plasma
}

\author{
Yue Liu ${ }^{1}$, Wei-Min Wang ${ }^{2}$, and Zheng-Ming Sheng ${ }^{1,3}$ \\ ${ }^{1}$ Key Laboratory for Laser Plasmas (MoE) and Department of Physics and Astronomy, Shanghai Jiao Tong University, \\ Shanghai, China \\ ${ }^{2}$ Beijing National Laboratory of Condensed Matter Physics, Institute of Physics, CAS, Beijing, China \\ ${ }^{3}$ Department of Physics, SUPA, Strathclyde University, Rottenrow 107, Glasgow, UK \\ (Received 14 February 2014; revised 14 February 2014; accepted 19 February 2014)
}

\begin{abstract}
It is demonstrated by simulations and analysis that a wakefield driven by an ultrashort intense laser pulse in underdense plasma can emit tunable electromagnetic radiation along the laser propagation direction. The profile of such a kind of radiation is closely associated with the structure of the laser wakefield. In general, electromagnetic radiation in the terahertz range with its frequency a few times the electron plasma frequency can be generated in the moderate intensity regime. In the highly nonlinear case, a chain of radiation pulses is formed corresponding to the nonlinear structure of the wake. Study shows that the radiation is associated with the self-modulation process of the laser pulse in the wakefield and resulting transverse electron momenta from modulated asymmetric laser fields.
\end{abstract}

Keywords: laser wakefield; PIC simulation; tunable radiation; transverse momentum

\section{Introduction}

Radiation generation in relativistic laser-plasma interaction has been a hot issue in the last two decades due to its wide application in medicine, biology, chemistry, etc. Nowadays, radiation over a wide range of frequency, from terahertz (THz) to $X / \gamma$, can be produced. Meanwhile, various scenarios of radiation generation have been developed, such as high-order harmonics generation ${ }^{[1-3]}$, betatron radiation $^{[4,5]}$, Thomson scattering ${ }^{[6]}$, Cerenkov radiation ${ }^{[7]}$, and linear mode conversion ${ }^{[8-10]}$. Each scheme of producing radiation sources has its own advantages and disadvantages in terms of pulse duration, spectral range, collimating property, and energy conversion efficiency. It is well known that the laser wakefield driven in homogeneous underdense plasma generally cannot produce radiation by itself. Only when the wakefield is excited in inhomogeneous plasma ${ }^{[9]}$ or in homogeneous plasma applied with an external DC magnetic field ${ }^{[11,12]}$, emission from laser wakefields can be generated. Recently, we showed that extremely ultraviolet (XUV) radiation can be produced from laser wakefields excited in homogeneous plasma ${ }^{[13]}$. The emission is caused by a transverse current sheet co-moving with the laser pulse, which is formed by an electron density spike with trapped

Correspondence to: Zheng-Ming Sheng, Key Laboratory for Laser Plasmas (MoE) and Department of Physics and Astronomy, Shanghai Jiao Tong University, 200240, No. 800 Dongchuan Road, Minhang District, Shanghai, China. Email: zmsheng@sjtu.edu.cn electrons in the wakefields together with certain transverse residual momentum of electrons left behind the laser pulse.

In this work, we extend our previous work on the wakefield emission in homogeneous plasma ${ }^{[13]}$ to a wider parameter range based upon numerical simulations. It is shown that electromagnetic radiation covering a wide frequency range from $\mathrm{THz}$ to XUV can be produced simultaneously with the laser wakefield excitation in underdense homogeneous plasmas. The strength and frequency of the radiation change significantly with the laser or plasma parameters. In the case with a weakly relativistic driving laser, the radiation produced turns out to be a multi-cycle $\mathrm{THz}$ pulse. If one increases the plasma density and the laser intensity, the radiation shows up in the form of a pulse chain with frequency around the laser frequency. As the laser intensity is enhanced further, wake wave-breaking occurs and XUV radiation is emitted ${ }^{[13,14]}$. A simple theoretical analysis is presented for the physical mechanisms involved.

\section{Wake radiation from one-dimensional PIC simula- tions}

To illustrate the emission from laser wakefield excitation, a series of one-dimensional (1D) particle-in-cell (PIC) simulations were conducted with the code $\mathrm{KLAP}^{[15]}$ and OSIRIS $2.0^{[16]}$. We take a laser pulse whose envelope in the form $a_{L}=a \sin ^{2}(\pi t / \tau)$, where $a=\sqrt{I \lambda_{L}^{2} /\left(1.37 \times 10^{18} \mathrm{~W} \mu \mathrm{m}^{2} \mathrm{~cm}^{2}\right)}$, 
(a)
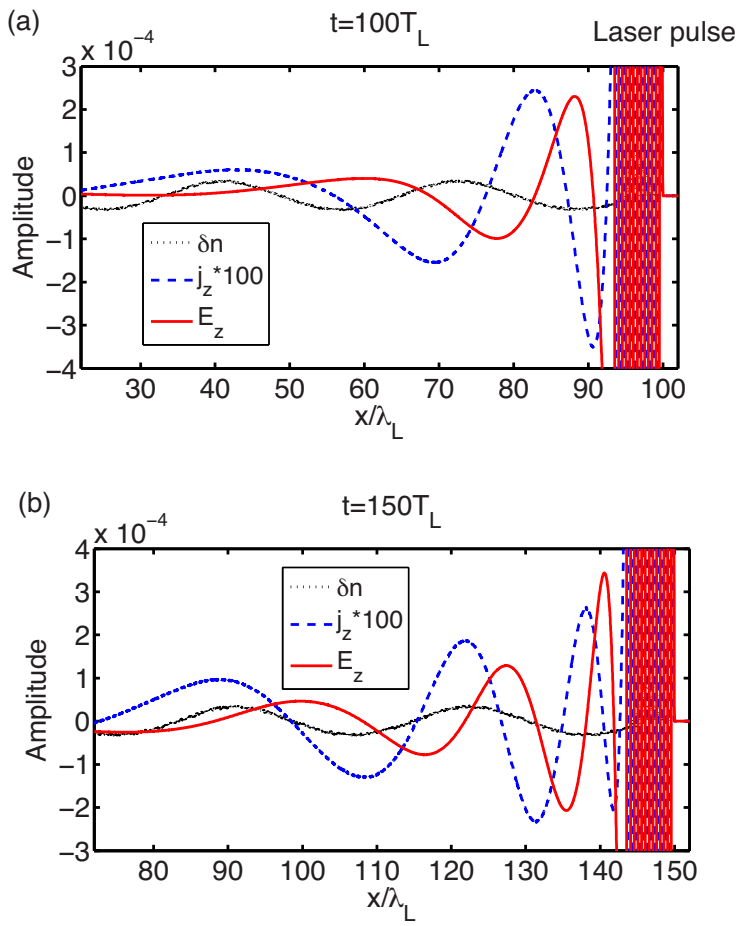

Figure 1. (Color online) The electromagnetic radiation waveform (red solid lines) in the wake at time $t=100 T_{L}$ and $t=150 T_{L}$; snapshots of the transverse current densities (blue dashed lines) and the electron densities (black dotted lines) are also plotted, where $a=0.5, n_{0}=0.0001$. The filled red region indicates the incident laser.

with $I$ the peak laser intensity. We also take $\lambda_{L}=1 \mu \mathrm{m}$, and $\tau=7 T_{L}$ is the full laser duration, with $T_{L}$ the laser oscillation period. It is assumed that the laser pulse is linearly polarized along the $z$-direction and is normally incident on a uniform plasma slab with density $n_{0}=n_{e} / n_{c}=0.001$ (corresponding to the electron plasma frequency $\omega_{p} / 2 \pi=$ $9.4 \mathrm{THz}$ ) along the $x$-direction, where $n_{c}$ is the critical density. The left vacuum-plasma boundary is set at $x=20 \lambda_{L}$. The length of the plasma slab depends on the laser propagation distance, and generally it varies between $100 \lambda_{L}$ and $200 \lambda_{L}$ in the simulation according to different cases. Simulations were conducted with different time and space resolutions to ensure that the results were not a numerical artefact.

Figure 1 displays the wake radiation waveform travelling forward in the wake right behind the laser pulse and the corresponding spatial distribution of the density perturbation (denoted by $\delta n=n-n_{0}$ ). The corresponding transverse current associated with the radiation is also shown. The frequency and the amplitude of the radiation behind the laser pulse are found to be different for each cycle, i.e., both the frequency and amplitude decrease with the distance from the driving laser pulse. At a later time $t=150 T_{L}$, there are more cycles of radiation formed. It is obvious that the amplitude of the wake radiation is larger at time $t=150 T_{L}$ than that at $t=100 T_{L}$. The wake radiation is found with the first cycle strongest. The radiation appears frequency chirped with
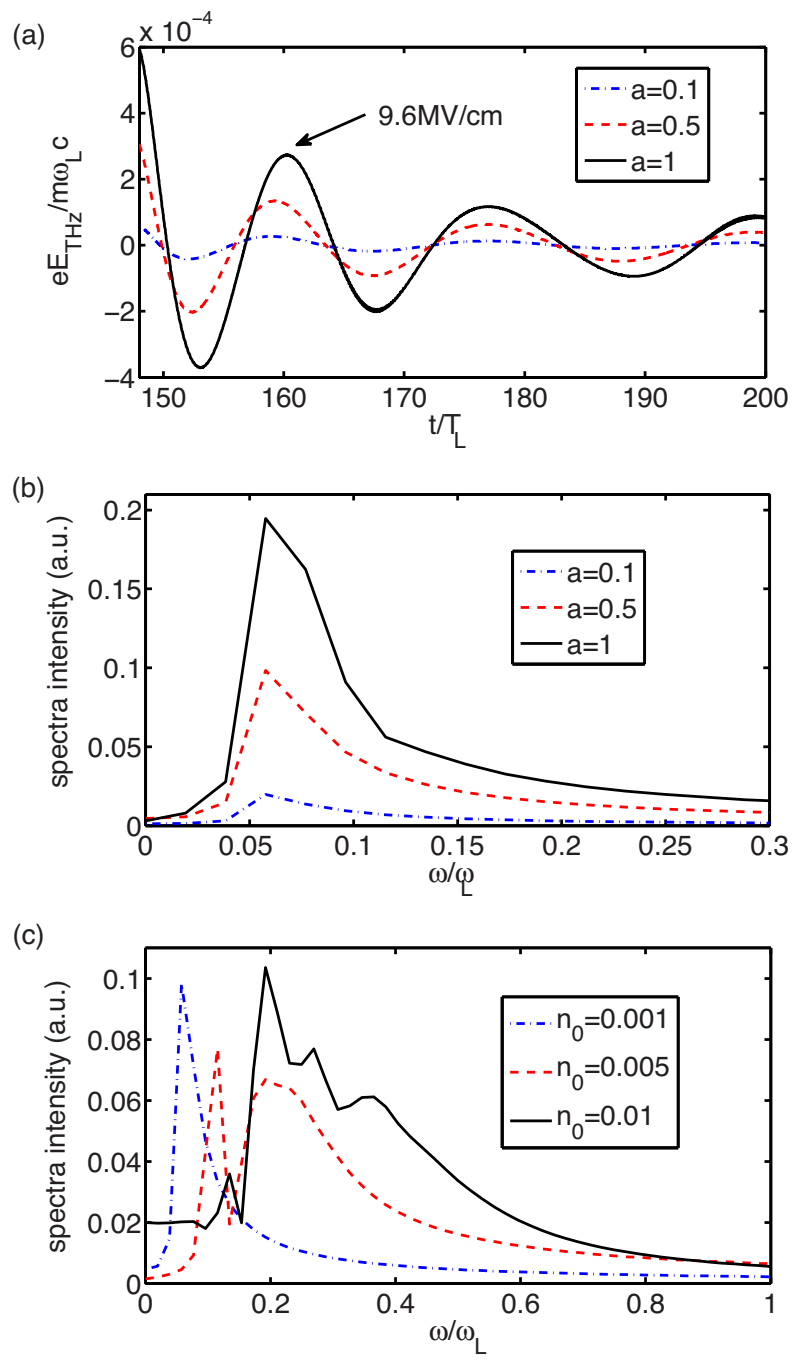

Figure 2. (Color online) The temporal waveforms of the radiation pulses observed at a fixed position at the right side of the simulation box in vacuum for three different laser intensities with initial plasma density $n_{0}=0.001$ (a) and the corresponding frequency spectra (b). Frequency spectra of the radiation produced with different plasma densities at a given laser amplitude $a=0.5$ are also plotted in (c).

the high-frequency component at the front, which remains unchanged during the whole process. This can be understood partially as due to plasma dispersion, such that the highfrequency component propagates at a higher speed.

For plasmas with $n_{0}=n_{e} / n_{c}=0.001$, the wake radiation turns out to be in the $\mathrm{THz}$ frequency regime. We show the temporal evolution of the radiation observed at the right side of the plasma slab in vacuum for three typically different laser intensities and their corresponding spectra in Figures 2(a) and 2(b). The radiation penetrates through the plasma into the vacuum following the laser pulse and has a waveform with the first cycle strongest, as mentioned above. In Figure 2(a), the THz radiation amplitude is found to be up to $9.6 \mathrm{MV} \mathrm{cm}^{-1}$ for the incident laser at $\sim 10^{18} \mathrm{~W} \mathrm{~cm}^{-2}$. However, the $\mathrm{THz}$ pulse frequency changes weakly with 


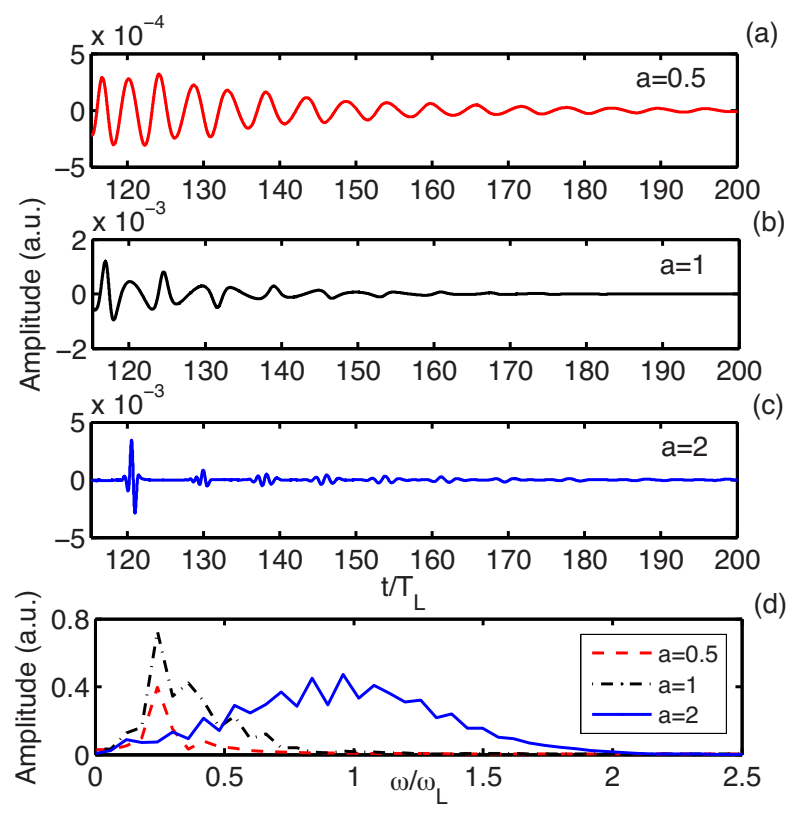

Figure 3. (Color online) The temporal evolution of the electromagnetic radiation emitted in the wake at three different laser intensities $(\mathrm{a}-\mathrm{c})$ and the corresponding frequency spectra (d), where $n_{0}=0.02$.

the laser amplitude in Figure 2(b) in the moderate intensity regime. In this case the central frequency of the $\mathrm{THz}$ pulses is about $18.9 \mathrm{THz}$. In addition, if we increase the plasma density, the radiation frequency is also shown to increase. As plotted in Figure 2(c), by increasing the plasma density from $0.001 n_{c}$ to $0.01 n_{c}$, the frequency spectrum is broadened, and its central frequency grows from $0.0625 \omega_{L}$ to $0.1875 \omega_{L}$. This implies that the radiation frequency increases with increasing plasma density. Note that these frequencies are also larger than the corresponding electron plasma frequencies. In the $n_{0}=0.005$ and $n_{0}=0.01$ cases, in addition to the main peaks, there are also other subpeaks in the spectra.

Next, we increase laser intensity further. Figure 3 shows the radiation propagating forward in the right side of the simulation box in vacuum for three different incident laser intensities in plasma with initial density $n_{0}=0.02$, as well as the corresponding frequency spectra. At weakly relativistic laser intensity, the radiation displays similar temporal waveforms as shown above. But at higher intensity for $a \geqslant 1$, the radiation shows up in the form of a pulse train. This appears to be associated with the highly nonlinear features of the wakefield excitation. Note that, with the increase of the laser intensity, the separation between neighbouring subpulses also increases. This agrees with the fact that the wavelength of a nonlinear plasma wave increases with its amplitude due to the relativistic-mass increase of electrons. Similar to the case of $n_{0}=0.001$, the central frequency of the radiation is shown not to change a lot for different laser intensity in the weakly relativistic regime, even for plasma density $n_{0}=0.02$ in Figure 3(d). In the case of $a=2$, the radiation frequency is approaching the laser frequency.
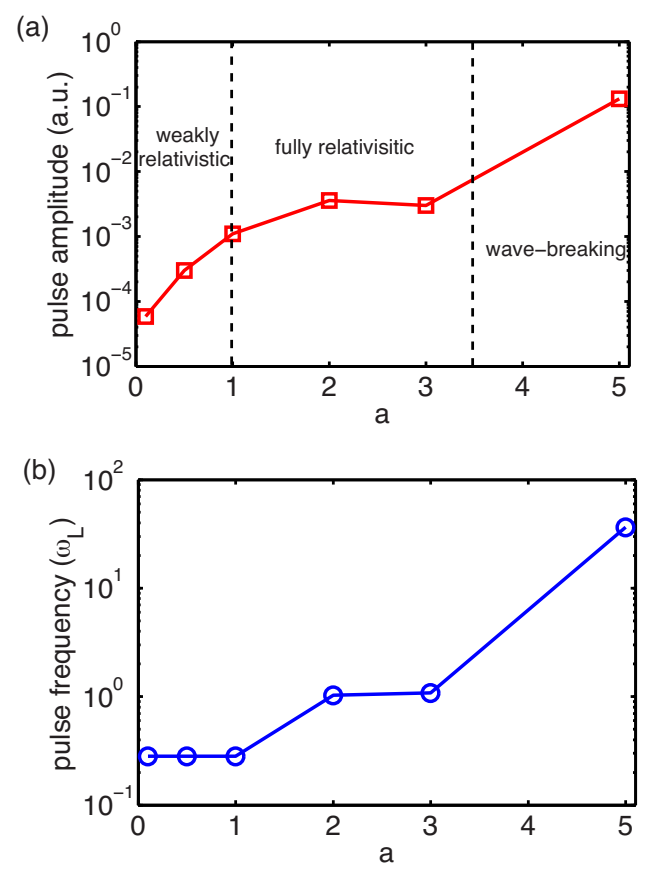

Figure 4. (Color online) The amplitude of the first pulse normalized by $m \omega_{L} c / e$ in the wake radiation (a) and its central frequency (b) versus the normalized laser strength $a$, where $n_{0}=0.02$.

Figure 4 illustrates the amplitude of the wake radiation in its first cycle behind the laser pulse and its central frequency as a function of the normalized laser vector potential. In non-relativistic and weakly relativistic regimes, the radiation amplitude scales with the laser intensity following the power law, as shown in Figure 4(a), whereas it generally changes little with the laser intensity in the relativistic regime. When the laser intensity is high enough that wave-breaking occurs in the driven wakefield, electron injection and trapping in the first wave-bucket are found. Thus the intensity of the emitted radiation is strengthened remarkably. As shown in Figure 4(a), the field for $a=5$ is about 20 times that for $a=3$. This is the regime which we have identified before ${ }^{[13,14]}$. Likewise, the frequency spectrum differs significantly for the weakly relativistic, fully relativistic, and the wave-breaking regimes, as illustrated in Figure 4(b). The central frequency of the radiation pulse remains nearly the same in the weakly relativistic regime, which confirms the previous results in Figure 2(b) under a different plasma density. In the fully relativistic regime, the radiation frequency becomes close to the laser frequency. In the wave-breaking regime, when the laser amplitude is enhanced to $a=5$, the central frequency approaches $50 \omega_{L}$ in the XUV regime.

\section{Two-dimensional PIC simulations}

To illustrate the multi-dimensional effects on the wake emission, we performed 2D PIC simulations. The 2D simulation box size is $50 \mu \mathrm{m} \times 50 \mu \mathrm{m}$, with 2500 cells along the laser propagation axis in the $x$-direction and 1000 cells along the 

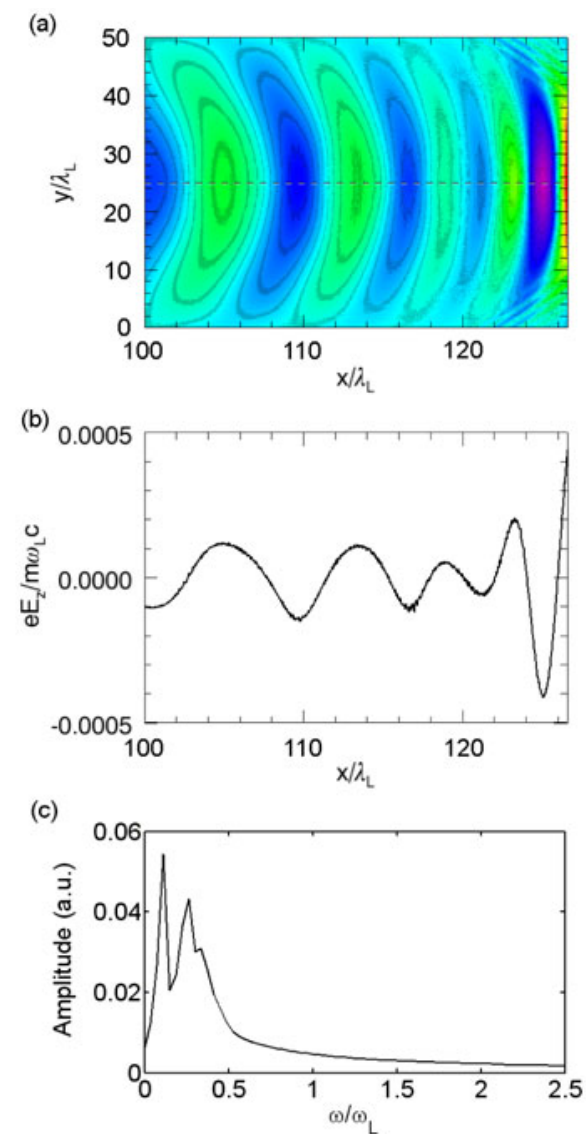

Figure 5. (Color online) 2D simulation results of wake radiation generation: (a) spatial distribution of electromagnetic radiation, (b) waveform of the radiation on the laser axis, and (c) frequency spectrum of the wake radiation, where $a=0.5$ and $n_{0}=0.02$.

$y$-direction. The driving laser pulse has a Gaussian profile in the transverse direction with $10 \lambda_{L}$ waist radius at the focal plane, and it is polarized in the $z$-direction with peak amplitude $a=0.5$. The laser pulse enters plasmas with density $n_{0}=0.02$ at $t=20 T_{L}$, and we choose the same plasma parameters as the above 1D simulation in Figure 4. Figure 5 shows the wake radiation in the laser wakefields behind the driving laser and its transverse field on the laser axis, as well as the corresponding frequency spectrum. Study shows that the waveform of the radiation is consistent with the results found in the 1D simulation. In addition, the amplitude and the frequency of the radiation here are both similar to the results of 1D simulations (as shown in Figure 3). Generally, the wake radiation tends to decay more quickly in the $2 \mathrm{D}$ case. This is due to the spatial transverse spreading effect of the electromagnetic waves when the radiation source size is comparable to the radiation wavelength.

\section{Theoretical model and discussions}

In previous work, we proposed a simple model for XUV emission in the wakefields ${ }^{[13]}$. Here, we consider the case

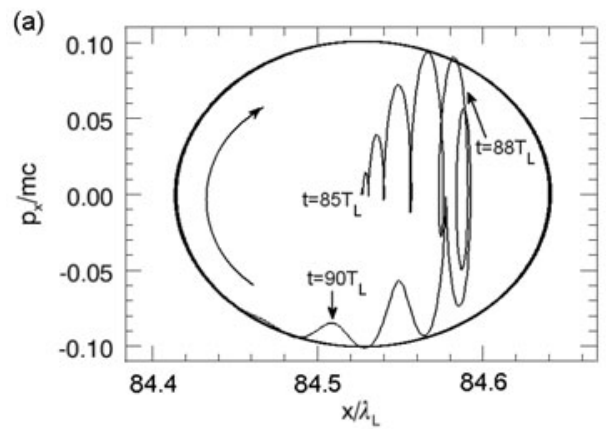

(b)

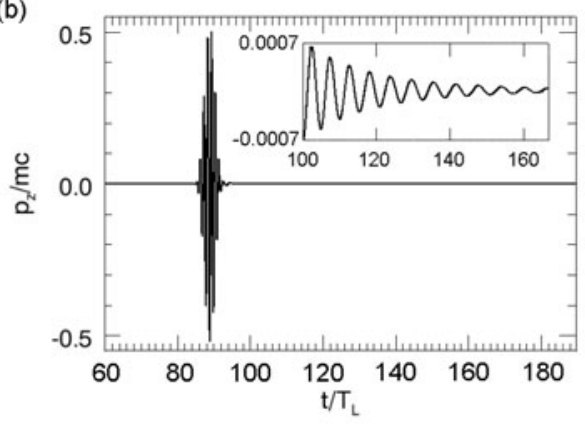

Figure 6. The trajectory of a typical electron during and after the laser interaction in phase space (a) and the temporal evolution of its transverse momentum (b), where $a=0.5, n_{0}=0.02$. The inset in (b) shows the transverse momentum at a later time after the laser interaction.

for a weakly relativistic intense laser pulse passing through underdense plasmas. The key issue is to understand the mechanism of the transverse current formation, which is responsible for the wakefield emission. For the case with an ultrashort few cycle laser pulse with its waveform highly asymmetric, it has been shown that a slowly varying transverse current can be generated behind the laser pulse ${ }^{[17]}$, which can produce radiation at the electron plasma frequency through the vacuum-plasma boundary. In the present case, however, the radiation is produced inside the homogeneous plasma, and its frequency is higher than the electron plasma frequency. Therefore, other physical processes may be involved.

Let us first check out a typical electron trajectory during and after the laser interaction. As illustrated in Figure 6(a), the electron is initially located at $x=84.54 \lambda_{L}$. During the laser interaction, it starts to move forward until it is at about $x=84.59 \lambda_{L}$, when it starts to move backward due to the charge separation field and the decreasing laser amplitude in its trailing edge. Finally, when the laser pulse passes by, it simply oscillates along the longitudinal direction in the established laser wakefield between $x=84.42 \lambda_{L}$ and $x=$ $84.64 \lambda_{L}$. Figure $6(\mathrm{~b})$ displays the transverse momentum of the electron during and after the laser interaction. After the laser interaction, a small transverse momentum is left. Note that the transverse momentum decays with time according to Figure 6(b). Correspondingly, the emitted radiation is also the strongest just behind the laser pulse, which agrees with 
the simulation results shown above. This transverse momentum oscillates much more slowly with time as compared with the laser frequency. Such oscillations occur after the laser pulse interacts with the electrons in the plasma. The net transverse momentum can be attributed to evolution of the laser pulse into slight asymmetry. Alternatively, the slowly varying transverse moment of electrons can also be attributed to self-modulation of the laser pulse. During this process, its carrier phase changes with time so that the electrons obtain net transverse acceleration at a certain time.

In the case when the driving laser is at fully relativistic intensity, the wakefield becomes highly nonlinear and has high-density peaks. The resulting radiation then appears in the form of a pulse chain with the subpulse separated by a plasma wavelength. This can explains the results shown in Figure 3(c). On either increasing the propagation distance or increasing the plasma density, simulation shows that the transverse momentum of electrons tends to increase because stronger modulation of the laser pulse may occur. This usually results in enhanced radiation, as shown in the simulation.

\section{Conclusion}

It is shown that, when an intense laser pulse propagates through homogeneous underdense plasma, tunable radiation in a wide frequency range can be produced in the wake of the laser pulse. Usually, it shows up with frequency higher than the electron plasma frequency and can transmit through plasma into vacuum following the driving laser pulse. $\mathrm{Nu}$ merical simulations indicate that this kind of electromagnetic emission occurs over a wide range of parameters for laser intensities and plasma densities. In the weakly relativistic regime, the wake emission turns out to be $\mathrm{THz}$ radiation in extremely tenuous plasmas. With increasing plasma density and laser intensity, the radiation appears in the form of a pulse chain corresponding to the wake structure. If the laser intensity is increased further, then wave-breaking occurs in the first plasma bucket and XUV radiation is produced. Qualitatively, the self-modulation of the laser pulse and the laser wakefield excitation are responsible for the emission. To reach a quantitative understanding, further study is required. This mechanism may provide a new possibility to produce a tunable coherent radiation for various applications.

\section{Acknowledgements}

This research was supported by the National Science Foundation of China (Grant No. 11121504, 11374209, 11374210, and 11375261). The authors also would like to acknowledge the OSIRIS Consortium, consisting of UCLA and IST (Lisbon, Portugal), for providing access to the OSIRIS 2.0 framework. The computational resources utilized in this research were provided partially by Shanghai Supercomputer Center.

\section{References}

1. P. Salieres, A. L'Huillier, and M. Lewenstein, Phys. Rev. Lett. 74, 3776 (1995).

2. E. J. Takahashi, Y. Nabekawa, and K. Midorikawa, Appl. Phys. Lett. 84, 4 (2004).

3. B. Dromey, S. G. Rykovanov, D. Adams, R. Hörlein, Y. Nomura, D. C. Carroll, P. S. Foster, S. Kar, K. Markey, P. McKenna, D. Neely, M. Geissler, G. D. Tsakiris, and M. Zepf, Phys. Rev. Lett. 102, 225002 (2009).

4. S. Kiselev, A. Pukhov, and I. Kostyukov, Phys. Rev. Lett. 93, 135004 (2004).

5. S. Kneip, C. McGuffey, J. L. Martins, S. F. Martins, C. Bellei, V. Chvykov, F. Dollar, R. Fonseca, C. Huntington, G. Kalintchenko, A. Maksimchuk, S. P. D. Mangles, T. Matsuoka, S. R. Nagel, C. A. J. Palmer, J. Schreiber, K. Ta. Phuoc, A. G. R. Thomas, V. Yanovsky, L. O. Silva, K. Krushelnick, and Z. Najmudin, Nat. Phys. 6, 980 (2010).

6. J. Meyer-ter Vehn and H. C. Wu, Eur. Phys. J. D 55, 433 (2009).

7. C. D'Amico, A. Houard, M. Franco, B. Prade, A. Mysyrowicz, A. Couairon, and V. T. Tikhonchuk, Phys. Rev. Lett. 98, 235002 (2007).

8. Z. M. Sheng, H. C. Wu, K. Li, and J. Zhang, Phys. Rev. E 69, 025401 (2004).

9. Z. M. Sheng, K. Mima, J. Zhang, and H. Sanuki, Phys. Rev. Lett. 94, 095003 (2005).

10. F. Quere, C. Thaury, P. Monot, S. Dobosz, Ph. Martin, J. P. Geindre, and P. Audebert, Phys. Rev. Lett. 96, 125004 (2006).

11. Z. D. Hu, Z. M. Sheng, W. J. Ding, W. M. Wang, Q. L. Dong, and J. Zhang, J. Plasma Phys. 78, 421 (2012).

12. Z. D. Hu, Z. M. Sheng, W. M. Wang, L. M. Chen, Y. T. Li, and J. Zhang, Phys. Plasmas 20, 080702 (2013).

13. Y. Liu, Z. M. Sheng, J. Zheng, F. Y. Li, X. L. Xu, W. Lu, W. B. Mori, C. S. Liu, and J. Zhang, New J. Phys. 14, 083031 (2012).

14. Y. Liu, F. Y. Li, M. Zeng, M. Chen, and Z. M. Sheng, Laser Part. Beams 31, 233 (2013).

15. M. Chen, Z. M. Sheng, J. Zheng, Y. Y. Ma, and J. Zhang, Chin. J. Comput. Phys. 25, 50 (2008).

16. R. A. Fonseca, L. O. Silva, F. Tsung, V. K. Decyk, W. Lu, C. Ren, W. B. Mori, S. Deng, S. Lee, T. Katsouleas, and J. C. Adam, Lect. Notes Comput. Sci. 2331, 342 (2002).

17. W. M. Wang, S. Kawata, Z. M. Sheng, Y.-T. Li, and J. Zhang, Phys. Plasmas 18, 073108 (2011). 